\title{
Neuroprotective Activity of (-)-Epigallocatechin Gallate against Lipopolysaccharide-Mediated Cytotoxicity
}

\author{
Jin-Biao Liu, ${ }^{1}$ Li Zhou, ${ }^{1}$ Yi-Zhong Wang, ${ }^{2}$ Xu Wang, ${ }^{2}$ Yu Zhou, ${ }^{2}$ \\ Wen-Zhe Ho, ${ }^{1,2,3}$ and Jie-Liang $\mathrm{Li}^{2}$ \\ ${ }^{1}$ Animal Biosafety Level III Laboratory at the Center for Animal Experiment, Wuhan University School of Basic Medical Sciences, \\ Wuhan 430071, China \\ ${ }^{2}$ Department of Pathology \& Laboratory Medicine, Temple University Lewis Katz School of Medicine, Philadelphia, PA 19140, USA \\ ${ }^{3}$ State Key Laboratory of Virology, Wuhan University, Wuhan 430071, China
}

Correspondence should be addressed to Wen-Zhe Ho; wenzheho@temple.edu and Jie-Liang Li; jieliang.li@temple.edu

Received 20 January 2016; Revised 15 March 2016; Accepted 17 March 2016

Academic Editor: Oscar Bottasso

Copyright (C) 2016 Jin-Biao Liu et al. This is an open access article distributed under the Creative Commons Attribution License, which permits unrestricted use, distribution, and reproduction in any medium, provided the original work is properly cited.

\begin{abstract}
Lipopolysaccharide- (LPS-) mediated systemic inflammation plays a critical role in neurodegenerative diseases. The present study was conducted to evaluate the protective effects of epigallocatechin gallate (EGCG), the major component in green tea, on LPS-mediated inflammation and neurotoxicity. LPS treatment of macrophages induced expression of proinflammatory cytokines (TNF- $\alpha$, IL-1 $\beta$, and IL-6). However, EGCG pretreatment of macrophages significantly inhibited LPS-mediated induction of these cytokines. In addition, EGCG significantly diminished LPS-induced inflammatory cytokines in the peripheral mononuclear blood cells (PBMCs). Supernatant from EGCG-pretreated and LPS-activated macrophage cultures was found to be less cytotoxic to neurons than that from non-EGCG-pretreated and LPS-activated macrophage cultures. Furthermore, EGCG treatment of neurons could inhibit LPS-induced production of reactive oxygen species (ROS). Thus EGCG represents a potent and useful neuroprotective agent for inflammation-mediated neurological disorders.
\end{abstract}

\section{Introduction}

Inflammation plays a critical role in the immunopathogenesis of neurodegenerative diseases such as Parkinson's disease, multiple sclerosis, Alzheimer's disease, and HIV-associated dementia (HAD). Activation of microglia, the intrinsic macrophages in the central nervous system (CNS) [1], is a characteristic feature of neurodegenerative diseases. Mounting evidence clearly indicates that macrophage/microglia activation contributes to inflammation and neuronal injury in the CNS $[2,3]$. Lipopolysaccharide (LPS), a major element of Gram-negative bacteria, is a potent activator of immune cells, particularly macrophages and microglia, as it induces expression of proinflammatory cytokines such as TNF- $\alpha$, IL6 , and IL-1 $\beta[4,5]$. These cytokines have direct or indirect neurotoxic effects on neuronal cells, causing neuronal injury. Microglial activation by LPS plays an important role in the progressive and selective loss of dopaminergic (DA) neurons
$[6,7]$. Microglia-derived superoxide contributes to about $50 \%$ of LPS-induced DA neurotoxicity $[8,9]$.

Although microglia are vital in the inflammatory process in the CNS, they may have less chance to be activated during a peripheral bacterial infection, as LPS may not be able to enter the CNS due to the blood-brain barrier (BBB). However, monocytes/macrophages in peripheral systems can become activated by LPS, which results in overexpression of proinflammatory cytokines. These cytokines can penetrate $\mathrm{BBB}$ and induce an inflammatory environment in the CNS [10]. In addition, activated monocytes in HIV infection have the ability to migrate into the CNS, causing neuronal injury [11]. Furthermore, exposure of macrophages/microglia to invading pathogens leads to the production of ROS, which can benefit the clearance of pathogens but on the other hand cause irreparable damage to neurons [12].

Natural products and dietary components rich in polyphenols have been regarded as promising dietary agents 
for the prevention and treatment of inflammation-related diseases [13]. (-)-Epigallocatechin gallate (EGCG) is the most abundant catechin in green tea, a beverage widely consumed worldwide. EGCG as a potent antioxidant has been shown to have both anti-inflammatory and antiatherogenic properties in experimental studies conducted in vitro and in vivo $[14,15]$. EGCG was found to inhibit TNF- $\alpha$-induced production of MCP-1/CCL2 from bovine coronary artery endothelial cells, providing direct vascular benefits in inflammatory cardiovascular diseases [16]. It has also been shown that EGCG attenuated the increase in malondialdehyde levels caused by cerebral ischemia and reduced the formation of postischemic brain edema and infarct volume [17]. The neuroprotective effect of EGCG against ischemia-induced brain damage was found, in part, due to the modulation of NOS isoforms and preservation of mitochondrial complex activity and integrity [18]. Thus, the in vivo neuroprotective effects of EGCG are not exclusively due to its antioxidant effects but involve more complex signal transduction mechanisms. In addition, the dose of EGCG is vital to be concerned in neuroprotective application, as EGCG presents a biphasic effect based on its concentration-dependent window of pharmacological action. EGCG can act as an antioxidant, reducing ROS at low concentrations [19, 20], and paradoxically may promote the production of ROS and decline of mitochondrial membrane potential and induce apoptosis at high concentrations [21]. In this study, we examined whether EGCG possesses the ability to protect primary human neurons from the macrophages-mediated inflammation and neurotoxicity.

\section{Materials and Methods}

2.1. (-)-Epigallocatechin Gallate. EGCG ( $\geq 95 \%)$ was purchased from Sigma-Aldrich, St. Louis, MO, USA (CAS\# 98951-5). EGCG stock solution was prepared in sterile double distilled water at $20 \mathrm{mM}$.

2.2. Endotoxin-Induced Inflammatory Response and EGCG Treatment. All animal experiments were conducted according to the guidelines for the care and use of laboratory animals and the protocols were approved by the Institutional Animal Care and Use Committee (IACUC) of Animal Biosafety Level III Laboratory at the Center for Animal Experiment. Sixteen adult male Sprague-Dawley rats weighing 200-300 g were obtained from the Center for Animal Experiment, Wuhan University. Briefly, rats were intraperitoneally injected with LPS (from Escherichia coli, 055:B5, Invivogen; $1 \mathrm{mg} / \mathrm{kg} ; n=4$ ) or EGCG $(5 \mathrm{mg} / \mathrm{kg} ; n=4)$ or EGCG $(5 \mathrm{mg} / \mathrm{kg})$ plus LPS $(1 \mathrm{mg} / \mathrm{kg} ; n=4)$ in $0.1 \mathrm{~mL}$ of endotoxin-free phosphate buffered saline (PBS) or $0.1 \mathrm{~mL}$ of PBS $(n=4)$. After $24 \mathrm{~h}$, the rats were anesthetized with ketamine and xylazine. Blood samples were collected by cardiac puncture into heparinized syringes. The peripheral blood mononuclear cells (PBMC) were isolated by Ficoll density gradient centrifugation. Following centrifugation $(1500 \times \mathrm{g}, 30 \mathrm{~min}$, room temperature), PBMC located at the interface were harvested and washed with PBS and lysed with Tri Reagent for RNA extraction.
2.3. Monocyte-Derived Macrophage Cultures. Monocytes were obtained from the Path BioResource of the University of Pennsylvania School of Medicine. Blood samples were screened for common blood-borne pathogens and certified to be pathogen-free. Monocytes were isolated by elutriation; the purity of isolated monocytes was higher than 95\%. Freshly isolated monocytes were resuspended in DMEM supplemented with $10 \%$ fetal bovine serum (FBS), penicillin $(100 \mathrm{U} / \mathrm{mL})$, streptomycin $(100 \mu \mathrm{g} / \mathrm{mL})$, and $1 \%$ nonessential amino acids. Cells were cultured in 48-well plates (Corning CellBIND Surface, Corning Incorporated, Corning, NY) at $2.5 \times 10^{5}$ cells per well. The medium was half-changed every $48 \mathrm{~h}$. After culture for 7 days, monocytes differentiated into macrophages. Macrophages were incubated with different concentrations of EGCG $(0,10,20$, and $40 \mu \mathrm{M})$ for $24 \mathrm{~h}$ prior to the treatment with LPS for additional $6 \mathrm{~h}$ after which the medium was replenished and cultured for additional $24 \mathrm{~h}$. Supernatant collected from macrophage cultures was used to treat primary human neurons. The cytotoxicity of EGCG to macrophages was measured using a 3-(4, 5-dimethylthiazol2-yl)-2, 5-diphenyltetrazolium bromide (MTT) assay as previously described [22].

2.4. Primary Human Neuron Cultures. Highly enriched neuronal cultures were prepared as described previously [23]. All of the experimental protocols were reviewed and approved by the Institutional Review Board of the University of Minnesota Medical School. Briefly, 11- to 19-week-old fetal brain tissues of aborted fetuses (3 donors) obtained from the Human Embryology Laboratory (University of Washington, Seattle, WA, USA) were dissociated and resuspended in neural basal medium containing B-27 serum-free supplement (contains antioxidants) plus penicillin $(100 \mathrm{U} / \mathrm{mL})$ and streptomycin $(100 \mu \mathrm{g} / \mathrm{mL})$. Dispersed cells were plated onto collagen-coated plates $\left(5 \times 10^{5}\right.$ cells/well in 24 -well plate) or chamber slides $\left(4 \times 10^{5}\right.$ cells/well in 4 -well chambers $)$. On day 12 , these brain-cell cultures contained $\sim 70-80 \%$ neurons (stained by anti-NeuN or anti-MAP2 antibodies), 15$25 \%$ astrocytes (stained by anti-GFAP antibody), and 3-7\% microglial cells (stained by anti-CD68 antibody). For highly enriched neuronal cultures, cell cultures were treated with uridine $(33.6 \mu \mathrm{g} / \mathrm{mL})$ and fluorodeoxyuridine $(13.6 \mu \mathrm{g} / \mathrm{mL})$ on day 5 , followed by replacement with neural basal medium with B-27 serum-free supplement (contains antioxidants) on day 6 and every 4 days thereafter. Highly purified neuronal cultures contained $>95 \%$ neurons, $2-3 \%$ astrocytes, and 1-2\% microglial cells.

2.5. Reverse Transcription and Quantitative Real-Time PCR. Total RNA was extracted with Tri Reagent (Sigma-Aldrich) and quantitated by spectrophotometric analysis. Reverse transcription was performed using the AMV transcriptase and RNasin (Promega Co., Madison, WI, USA) according to the manufacturer's instruction. Quantitative realtime PCR (qRT-PCR) was performed with Brilliant SYBR Green Master Mix (Bio-Rad Laboratories, Hercules, CA, USA) described previously [24]. The oligonucleotide primers were synthesized by Integrated DNA Technologies, Inc. (Coralville, IA, USA). The primers that we used for the 


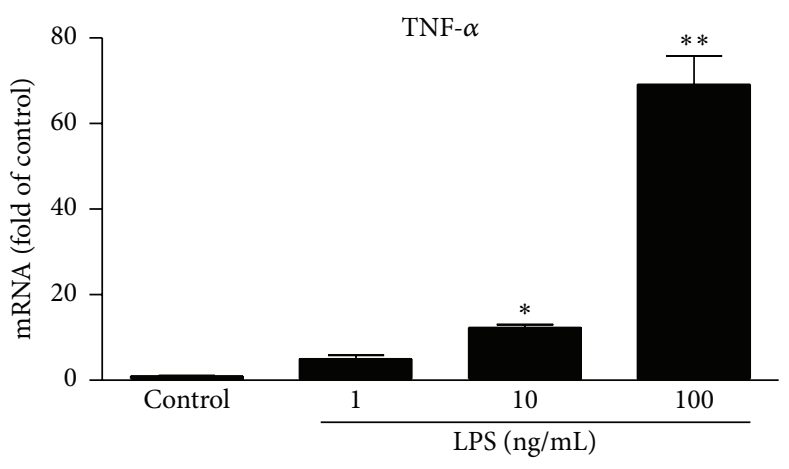

(a)

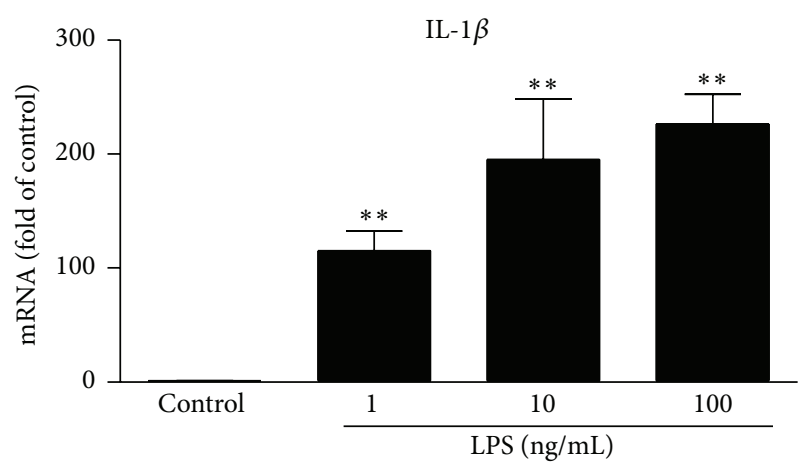

(b)

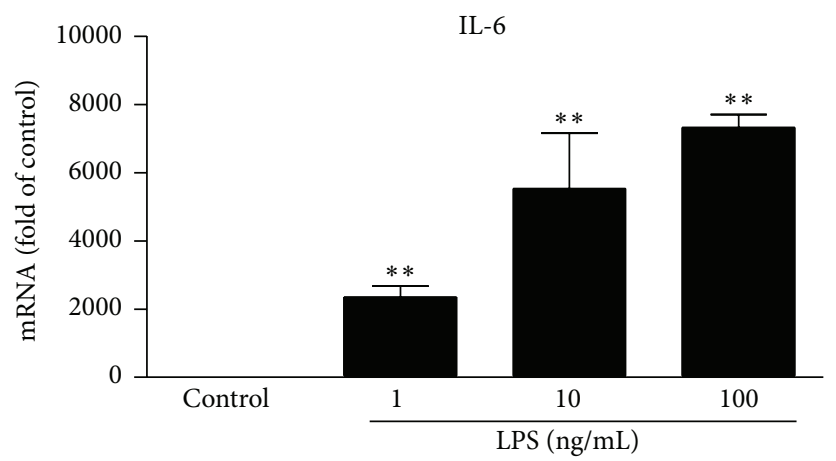

(c)

FIGURE 1: LPS induces the expression of inflammatory cytokines. Human macrophages derived from peripheral blood monocytes were treated with indicated concentrations of LPS for $24 \mathrm{~h}$. Cellular RNA was extracted and subjected to quantitative real-time RT-PCR for TNF- $\alpha$ (a), IL-1 $\beta$ (b), and IL-6 (c). Data were expressed as mean \pm SD of three independent experiments. Data were expressed as mean \pm SD of three independent experiments. ${ }^{*} P<0.05 ;^{* *} P<0.01$.

PCR amplifications are listed as follows: glyceraldehyde 3-phosphate dehydrogenase (GAPDH): 5'-GGTGGTCTCCTCTGA CTTCAACA- $3^{\prime}$ (sense) and $5^{\prime}$-GTTGCTGTAGCCAAATTCGTTGT- $3^{\prime}$ (antisense); TNF- $\alpha: 5^{\prime}$-CGAGTGACAAGCCTGTAGC- $3^{\prime}$ (sense) and $5^{\prime}$-GGTGTGGGTGAGGAGC ACAT- $3^{\prime}$ (antisense); IL-1 $\beta: 5^{\prime}$-AAGCTGATGGCCCTAAACAG- $3^{\prime}$ (sense) and $5^{\prime}$-AGGTGCATCGTGCACATAAG- ${ }^{\prime}$ (antisense); IL-6: $5^{\prime}$-AGGAGACTTGCCTGGTGA AA- $3^{\prime}$ (sense) and $5^{\prime}$-CAGGGGTGGTTATTGCATCT-3' (antisense); iNOS: $5^{\prime}$-GCAGAATGTGACCATCATGG- $3^{\prime}$ (sense) and $5^{\prime}$-ACAACCTTGGTGTTGAAGGC- $3^{\prime}$ (antisense). All values were calculated using the deltadelta cycle threshold method and expressed as the change relative to the expression of GAPDH.

2.6. Immunofluorescence Staining and MAP-2 ELISA. Neuronal cells were seeded on poly-L-lysine coated cover slips in 96-well plates and cultured for two weeks before treatment with LPS or supernatant from LPS-activated macrophage cultures. Cells were then washed with PBS three times and fixed in ice-cold methanol for $5 \mathrm{~min}$. Nonspecific sites were blocked in Block A for $30 \mathrm{~min}$. Cells were then incubated in mouse anti-MAP-2 antibody (1:100; Sigma-Aldrich, St. Louis, MO) for $1 \mathrm{~h}$, followed by Alexa 488 -conjugated antimouse IgG for $30 \mathrm{~min}$. After Hoechst $(2 \mu \mathrm{g} / \mathrm{mL})$ staining, the coverslips were mounted on glass slide and observed under a fluorescence microscope (Olympus IX71). For MAP2 ELISA, after block, cells were incubated with anti-MAP2 antibody $(1: 1000)$ overnight at $4^{\circ} \mathrm{C}$. After a wash with PBS, goat $\alpha$-mouse $\beta$-lactamase TEM-1 (Molecular Probes, Eugene, OR) conjugate $(1: 500 ; 2 \mu \mathrm{g} / \mathrm{mL})$ was added into each well and incubated for $30 \mathrm{~min}$ and then with fluorocillin green substrate (Invitrogen, Carlsbad, CA) solution in PBS $(1 \mu \mathrm{g} / \mathrm{mL})$ for $1 \mathrm{~h}$. Fluorescence was read at $485 / 527 \mathrm{~nm}$ on a SpectraMax ${ }^{\circledR}$ M3 Multi-Mode Microplate Reader (Molecular Devices, Sunnyvale, CA). The fluorescence of untreated neurons (control) was defined as $100 \%$.

2.7. Reactive Oxygen Species (ROS) Detection. Macrophages were pretreated with or without EGCG for $1 \mathrm{~h}$ prior to LPS treatment for $24 \mathrm{~h}$. Cells were then washed with serum-free medium and incubated in $10 \mu \mathrm{M}$ of $2^{\prime} 7^{\prime}$-dichlorofluorescin diacetate $\left(\mathrm{DCFH}_{2} \mathrm{DA}\right.$; Molecular Probes) at $37^{\circ} \mathrm{C}$ for $30 \mathrm{~min}$ [24]. After a counterstaining of nuclear with Hoechst 33342 $(2 \mu \mathrm{g} / \mathrm{mL})$ for $5 \mathrm{~min}$ and wash, the ROS production was assessed using a fluorescence microscope (Olympus IX71) at $488 / 527 \mathrm{~nm}$.

2.8. Statistical Analysis. Data are expressed as the mean \pm SD for at least three independent experiments. Statistical significance was analyzed using Student's $t$-test to compare the means of two groups. For comparison of means of 


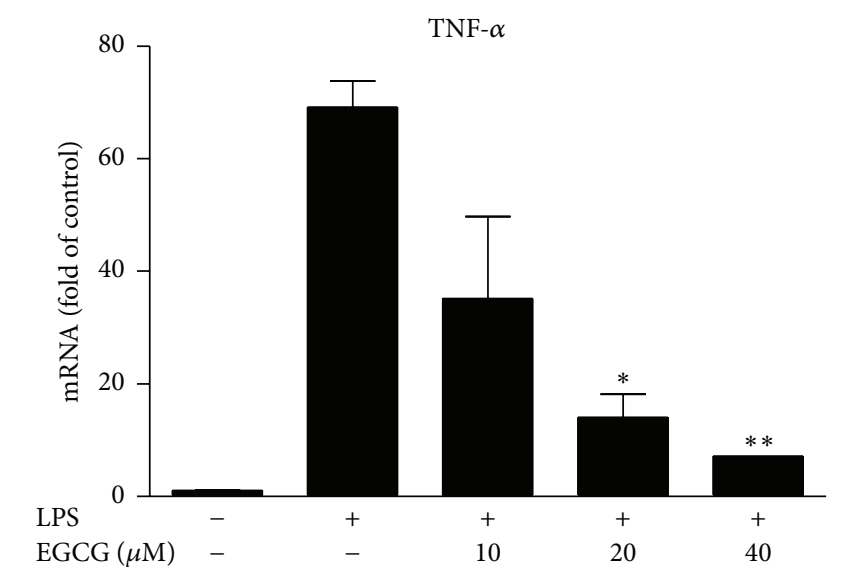

(a)

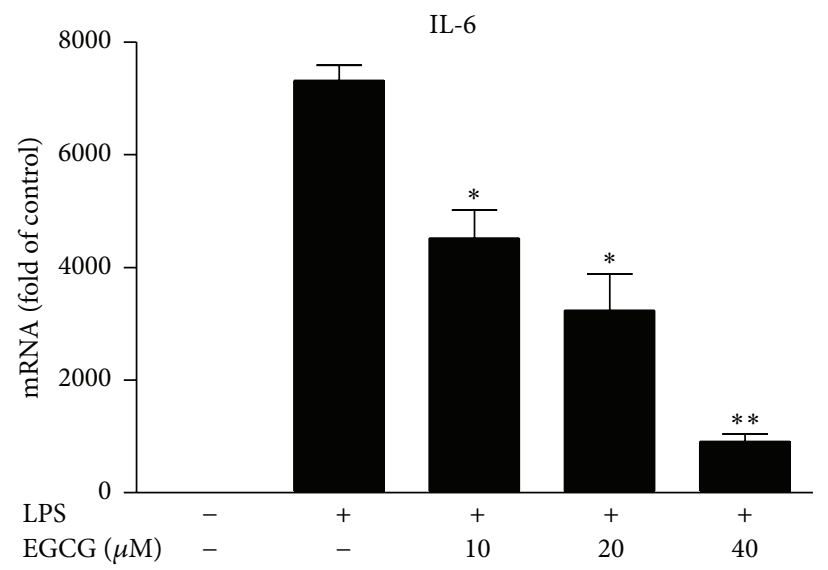

(c)

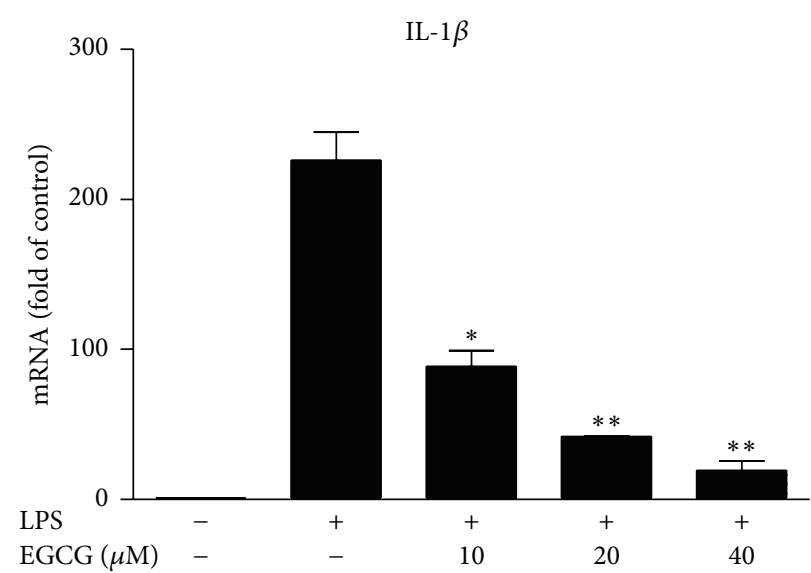

(b)

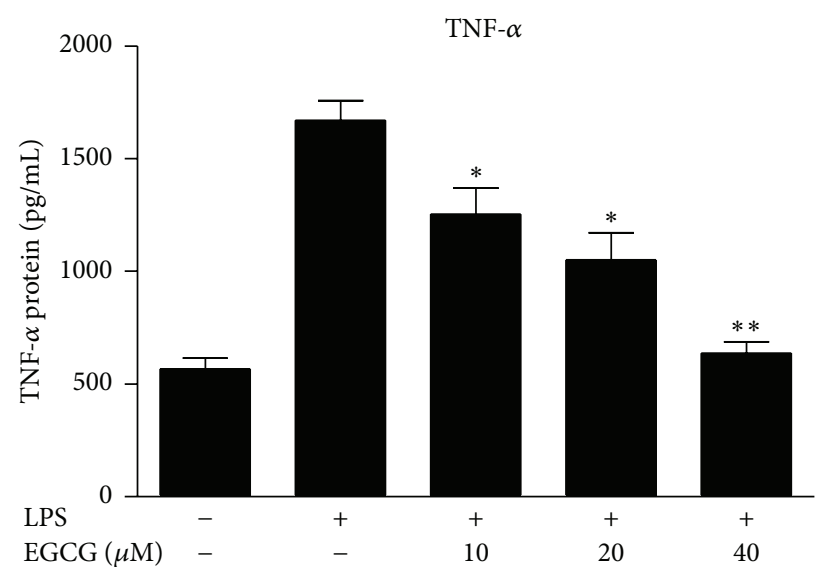

(d)

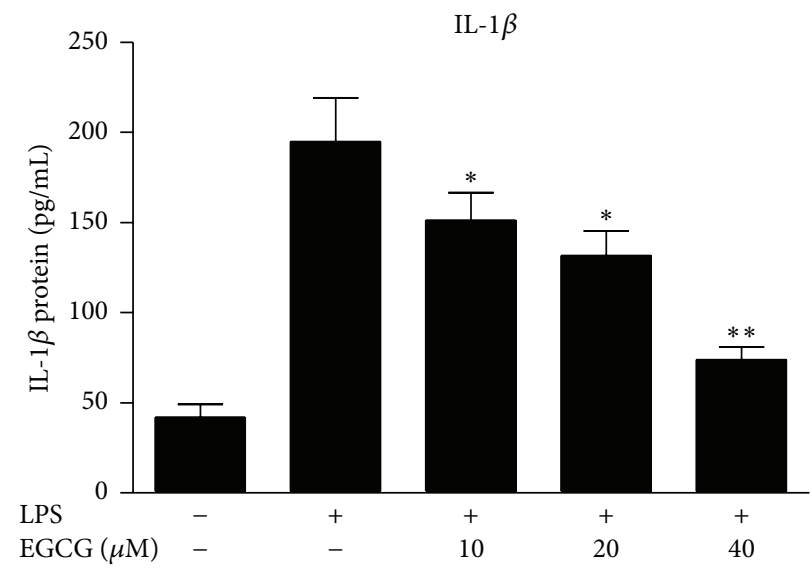

(e)

FIGURE 2: EGCG inhibits LPS-induced expression of inflammatory cytokines. Human macrophages derived from peripheral blood monocytes were treated with indicated concentrations of EGCG for $1 \mathrm{~h}$ prior to $100 \mathrm{ng} / \mathrm{mL}$ of LPS treatment for additional $24 \mathrm{~h}$. RNA was extracted and subjected to quantitative real-time RT-PCR of TNF- $\alpha$, IL- $1 \beta$, and IL-6. Data were expressed as mean \pm SD of three independent experiments. ${ }^{*} P<0.05 ;{ }^{* *} P<0.01$, as compared with LPS treated. 

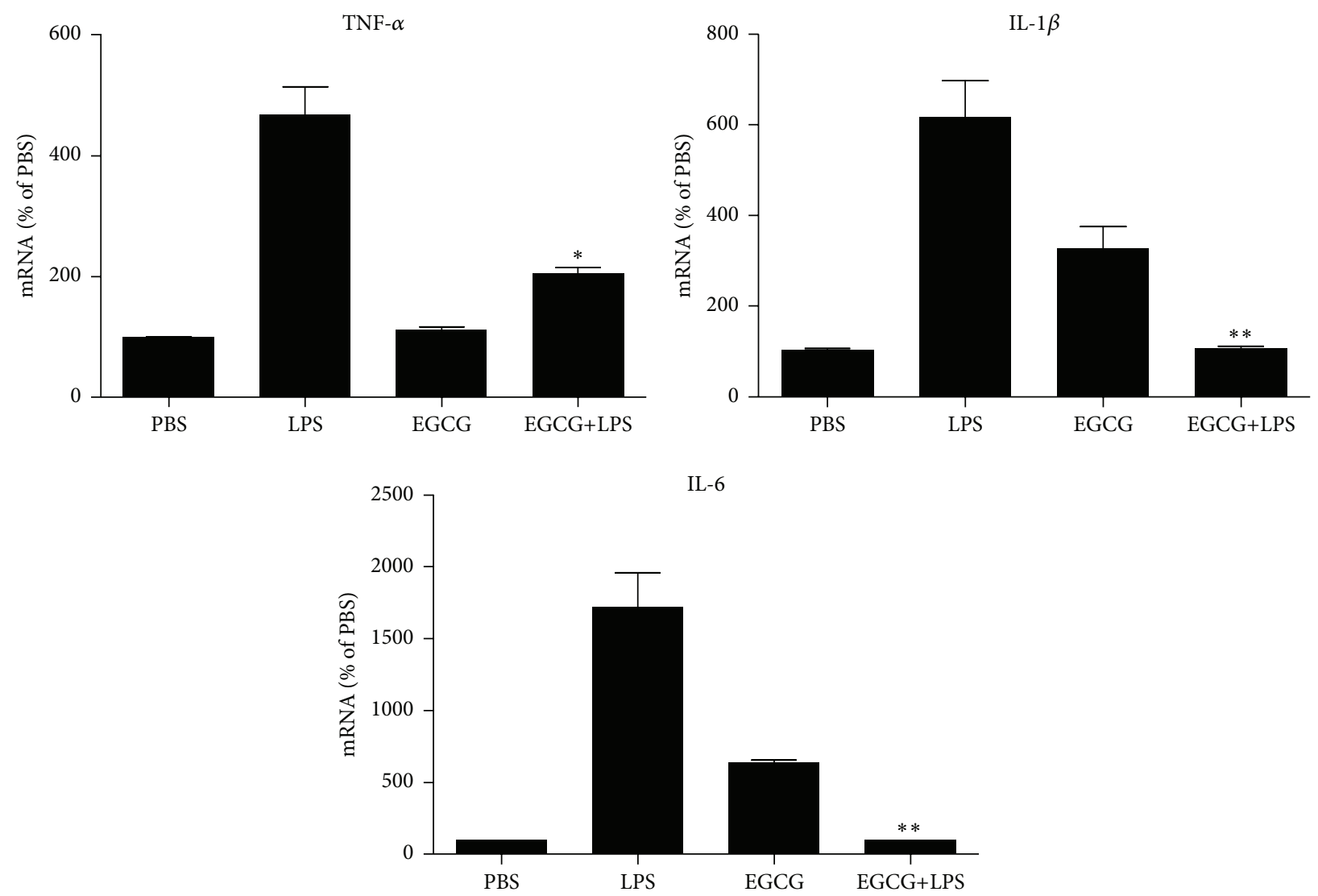

FIGURE 3: EGCG suppresses LPS-induced expression of inflammatory cytokines in rats. Sprague-Dawley rats were injected with PBS, LPS $(1 \mathrm{mg} / \mathrm{kg})$, EGCG $(5 \mathrm{mg} / \mathrm{kg})$, or LPS plus EGCG. Twenty-four hours posttreatment, rats $(n=4)$ in each group were sacrificed $24 \mathrm{~h}$ after being anesthetized. PBMCs were isolated by Ficoll-plaque and lysed with Tri Reagent. RNA was extracted and subjected to quantitative real-time RT-PCR for TNF- $\alpha$, IL-1 $\beta$, and IL-6. Data were expressed as mean \pm SD of 4 animals in each group. ${ }^{*} P<0.05 ;{ }^{* *} P<0.01$, as compared with LPS treated.

multiple groups, one-way analysis of variance (ANOVA) was performed followed by post-Newman-Keuls test. Differences were considered to be statistically significant when the $P$ value was less than 0.05 .

\section{Results}

3.1. EGCG Attenuates LPS-Induced Inflammatory Cytokines. We first evaluated the in vitro effects of EGCG on LPSinduced inflammatory cytokines in primary human macrophages. As shown in Figure 1, LPS treatment of macrophages induced the expression of TNF- $\alpha$, IL- $1 \beta$ (600-fold), and IL-6 (1700-fold). However, the expression of these cytokines were significantly reduced in macrophages pretreated with EGCG (Figure 2). This effect of EGCG was dose-dependent (Figure 2) without cytotoxicity (data not shown). We then examined the in vivo impact of EGCG on LPS-induced inflammatory cytokines in PBMCs of rats. As shown in Figure 3, LPS challenge of rats induced the expression of TNF- $\alpha$ (480-fold), IL-1 $\beta$ ( 600 -fold), and IL- 6 (1700-fold) in PBMCs. In contrast, EGCG administration significantly attenuated the induction of these cytokines by LPS (Figure 3).
3.2. Effect of EGCG on LPS-Induced Neurotoxicity through Macrophages. We next examined the protective effect of EGCG on LPS-induced neurotoxicity. Figure 4 shows that treatment of primary human neurons with supernatant from LPS-activated macrophage cultures significantly reduced the neuron numbers as identified by MAP-2 immunocytochemistry staining. However, EGCG pretreatment of macrophages remarkably inhibited LPS-induced neurotoxicity (Figure 4).

3.3. EGCG Protects Neurons from LPS-Induced Neurotoxicity. From the above we used macrophage cultures to mimic the microglia and we observed that supernatant from LPS-activated macrophages exerted neurotoxicity. Indeed, because it is difficult to obtain pure neuron population (even it can be $>95 \%$ ), there were microglia present in the neuronal cultures albeit at small numbers. We then examined whether LPS direct treatment of the neuronal cultures had neurotoxicity and whether EGCG could protect neurons in this context. When directly added to the neuronal cultures, LPS induced neurotoxicity as evidenced by the reduction of MAP-2 expression (Figure 5). Pretreatment of neurons with EGCG could protect neuronal cells from LPS-mediated cytotoxicity. However, the EGCG concentration $(0.1 \mu \mathrm{M})$ that 

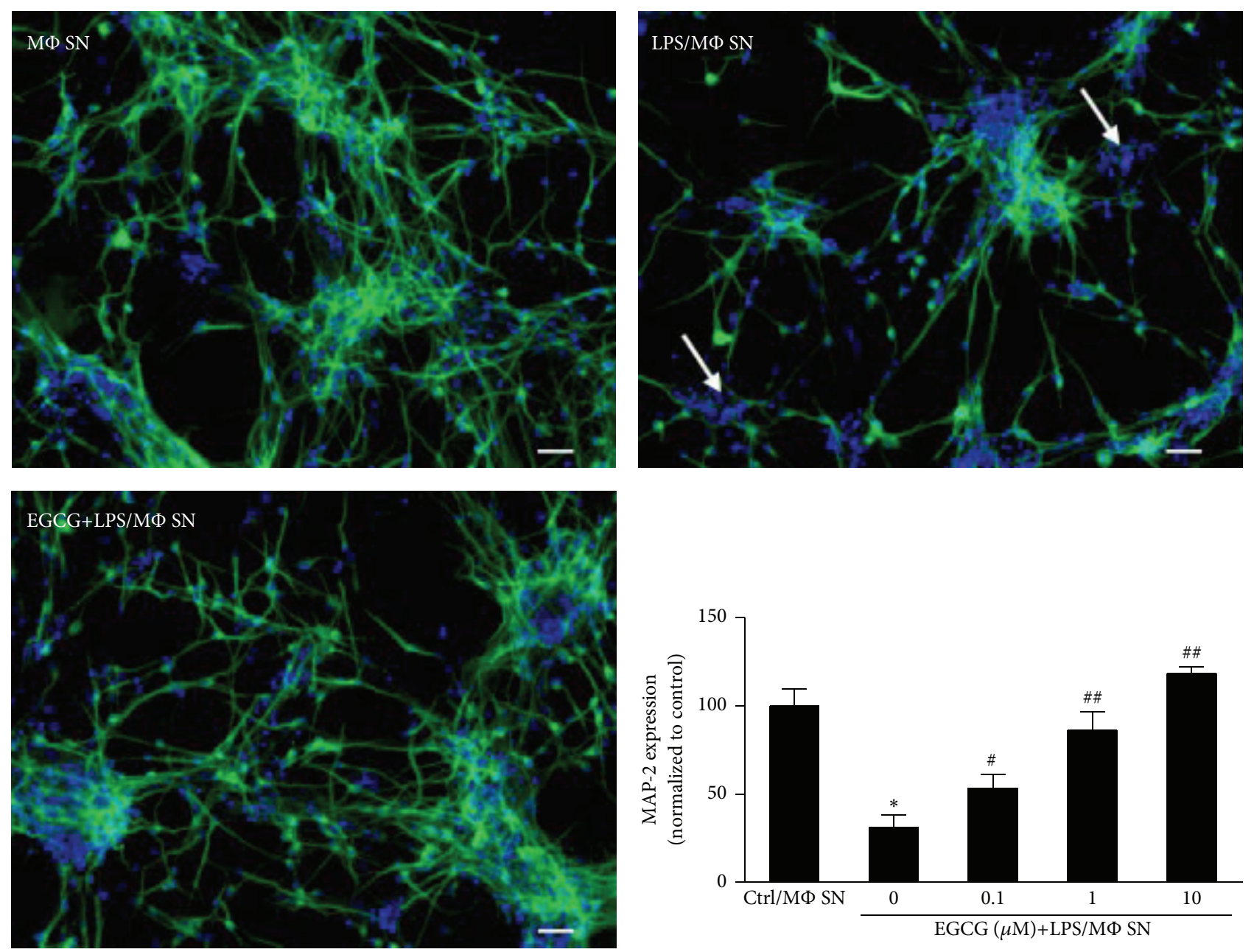

FIGURE 4: EGCG attenuates the neurocytotoxicity of supernatant from LPS-treated macrophage cultures to human primary neurons. Human macrophages derived from peripheral blood monocytes were treated with $100 \mathrm{ng} / \mathrm{mL}$ of LPS for $24 \mathrm{~h}$. For EGCG group, macrophages were pretreated with $10 \mu \mathrm{M}$ of EGCG for $1 \mathrm{~h}$. Culture supernatant from these macrophage cultures was used to treat human neurons (10\%, v/v). The neurocytotoxicity was examined by a cell-based MAP-2 ELISA. Nuclei were counterstained with Hoechst. Data were expressed as mean $\pm \mathrm{SD}$ and representative data from three independent experiments was shown. Magnification: $\times 100 .{ }^{*} P<0.05$, as compared with control; ${ }^{\#} P<0.05 ;{ }^{\# \#} P<0.01$, as compared with LPS treated. Scale bar $=50 \mu \mathrm{m}$.

can protect neurons directly is much lower than the effective concentrations $(10-40 \mu \mathrm{M})$ in protecting macrophages from LPS-induced upregulation of cytokines and macrophagesmediated neurocytotoxicity.

3.4. EGCG Inhibits LPS-Induced ROS Production in Neurons. To investigate the mechanism(s) of EGCG against LPSinduced direct neurocytotoxicity, we examined the oxidative stress in the neuronal cultures. ROS has been reported as an important mediator for LPS-induced cytotoxicity. Figure 6(a) shows that LPS treatment of neurons directly induced ROS production and this effect was dose-dependent. EGCG pretreatment inhibited LPS-mediated induction of ROS (Figure 6(b)), as well as the upregulation of iNOS (Figure $6(\mathrm{c}))$, but the EGCG concentration $(0.1 \mu \mathrm{M})$ was much lower than that required to exhibit the anti-inflammatory effect in macrophages $(10 \mu \mathrm{M})$.

\section{Discussion}

It is well known that activated macrophages or microglia produce inflammatory mediators, which has a negative impact on the survival of neurons $[1,25,26]$. Overactivated microglia/macrophages are a chronic source of multiple neurotoxic factors, including TNF- $\alpha$, NO, IL- $1 \beta$, and ROS that can cause progressive neuron damage [26-28]. We found that culture supernatant from LPS-stimulated macrophages exerted neurotoxicity to primary human neurons as evidenced by the reduced expression of specific neuronal marker MAP-2. Systemic inflammatory response which resulted from microbial infection is partly mediated by various pathogen-associated molecular patterns (PAMPs), such as endotoxin [29]. Bacterial endotoxin challenge or exposure plays an important role in inflammation-related damages, including neurodegeneration $[2,30]$. Although the production of proinflammatory cytokines (e.g., TNF- $\alpha$ and 


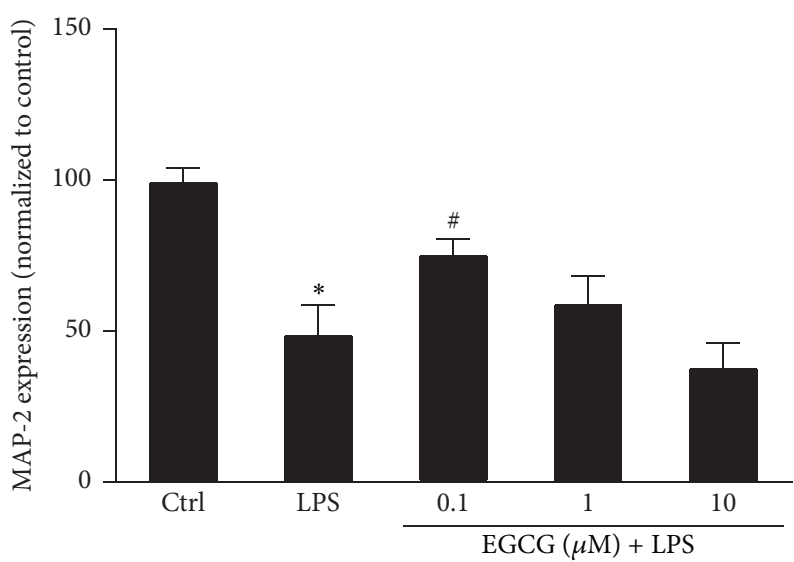

FIGURE 5: EGCG reduces neurocytotoxicity of LPS to neurons. Primary human neurons were treated with or without $0.1 \mu \mathrm{M}$ of EGCG for $1 \mathrm{~h}$ and then with $100 \mathrm{ng} / \mathrm{mL}$ of LPS for additional $48 \mathrm{~h}$. Cells were fixed and stained with anti-MAP-2. Nuclei were counterstained with Hoechst. Data were expressed as mean \pm SD of three independent experiments and representative figures were shown. ${ }^{*} P<0.05$, as compared with control; ${ }^{\#} P<0.05$, as compared with LPS only.

IL-6) by macrophages/microglia is essential in early host defense against infection [31], excessive accumulation of these cytokines disrupts systemic or CNS homeostasis [32-35]. EGCG has been shown to inhibit the induction of TNF$\alpha$ and IL- 6 in murine peritoneal macrophages elicited by TLR2/4 signaling $[4,36]$. Suppression of IFN- $\gamma$ and IL-6induced STAT signaling by EGCG has also been reported in mouse splenic monocytes and PBMCs [37, 38]. In addition, our earlier in vitro study showed that EGCG pretreatment of human brain microvascular endothelial cells could inhibit LPS-induced expression of inflammatory cytokines [39]. We found that in vivo EGCG administration to rat significantly reduced LPS-induced expression of proinflammatory cytokines (TNF- $\alpha$, IL- 6 , and IL-1 $\beta$ ) in PBMCs. The underlying mechanism(s) of the EGCG actions has largely been attributed to its suppression of NF- $\kappa$ B activation as well as the negative regulation of cytokine signaling [4, 38-41].

Green tea has been regarded as a nutrient component with possible beneficial effects on neurons although the cellular and molecular mechanism(s) remain unclear. EGCG is the main and most significantly bioactive polyphenol in green tea. We observed that EGCG inhibited the LPS-mediated induction of inflammatory cytokines and attenuated neurotoxicity by LPS-activated macrophages. In addition, EGCG at low dose $(0.1 \mu \mathrm{M})$ also exerted direct neuroprotective effect against LPS by mitigating the ROS production in neurons. These findings together with studies by others $[39,42,43]$ support the notion that EGCG has potential for treating inflammation-induced neuronal injury. Several reports indicated that tea polyphenols can be attained in the brain and exert neuroprotective effect simply by drinking [44-46]. EGCG metabolite could be detected in the brain after oral administration of EGCG to rats [47, 48]. An early observation that there was a wide distribution of labelled
EGCG in mouse organs including brain suggests the ability of EGCG to penetrate the BBB [49]. A single, very high oral EGCG dose $(500 \mathrm{mg} / \mathrm{kg}$ body weight) to rats yielded EGCG concentrations of about $0.5 \mathrm{nmol} / \mathrm{g}$ in brain (measured by CL-HPLC) and 20-fold higher in plasma [50]. EGCG was also investigated as a therapeutics adjuvant in the combination therapy to treat multiple sclerosis in mice [51]. However, due to limited systemic absorption, the concentrations of EGCG or EGCG metabolite in the brain are much lower than those in plasma [49].

Interestingly, we revealed that EGCG, at a lower dose of $0.1 \mu \mathrm{M}$, but not at higher concentrations ( 1 and $10 \mu \mathrm{M})$, protected neurons from LPS-induced direct neurotoxicity. This neuroprotective activity was concomitantly with the inhibition of ROS production by EGCG in LPS-treated neuronal cultures. Indeed, treatment of neurons with higher concentration $(10 \mu \mathrm{M})$ of EGCG increased ROS production (data now shown). This biphasic mode of antioxidant and prooxidant activities of EGCG has also been observed in other models $[52,53]$. It has been proposed that EGCG exhibits prooxidant and proapoptotic activity at high concentrations, which are responsible for its anticancer cell death effect, while lower doses of EGCG exert neuroprotection against a wide spectrum of neurotoxic compounds [54, 55]. Kucera et al. showed that low doses $(<10 \mu \mathrm{M})$ of EGCG decreased ROS production whereas EGCG in concentrations of $10 \mu \mathrm{M}$ and higher induced increase in ROS formation with resultant cellular injury and a decrease in hepatocyte functions. It was revealed that EGCG at high doses led to an uncoupling of mitochondrial oxidative phosphorylation and to damage to the outer mitochondrial membrane [19]. The oxidant activity of EGCG has also been demonstrated in murine macrophages and human leukemic cell lines to increased $\mathrm{H}_{2} \mathrm{O}_{2}$-induced oxidative stress and DNA damage $[56,57]$. Catechins, particularly EGCG $(100 \mu \mathrm{M})$, have been shown to increase the oxidative damage to isolated and cellular DNA after exposure to 8-oxo-7,8-dihydro-2' deoxyguanosine $[58,59]$. The prooxidant activity of EGCG was due to the generation of the hydroxyl radical and hydrogen peroxide in the presence of copper(II) and iron(III), suggesting that antioxidant mechanism of scavenging metals by catechins to stop the formation of free radicals may lead to prooxidant actions on DNA [60]. Excessive EGCG concentrations could also induce toxic levels of ROS in vivo. The prooxidative activities and dose-response relationship of EGCG have been implicated in the inhibition of lung cancer cell growth both in vivo and in vitro [21]. In our in vivo experiment, we noticed that EGCG treatment of rat also slightly induced the upregulation of IL- $1 \beta$ and IL-6, which might attribute to the prooxidant activity of EGCG. This concentration-dependent biphasic mode is common for some typical radical scavengers and antioxidants, such as ascorbic acid (vitamin C) [61].

In summary, we provide experimental evidence that EGCG attenuates LPS-induced inflammation and LPSactivated macrophage-mediated neurotoxicity at relative higher concentrations $(10-40 \mu \mathrm{M})$. EGCG at low dose $(0.1 \mu \mathrm{M})$, but not high concentrations used in macrophages, protects neurons from LPS-induced neurotoxicity and the 


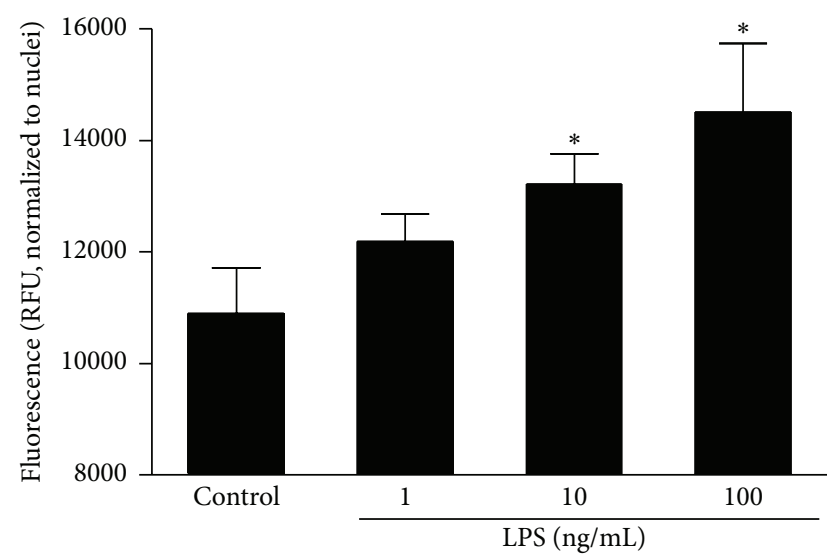

(a)

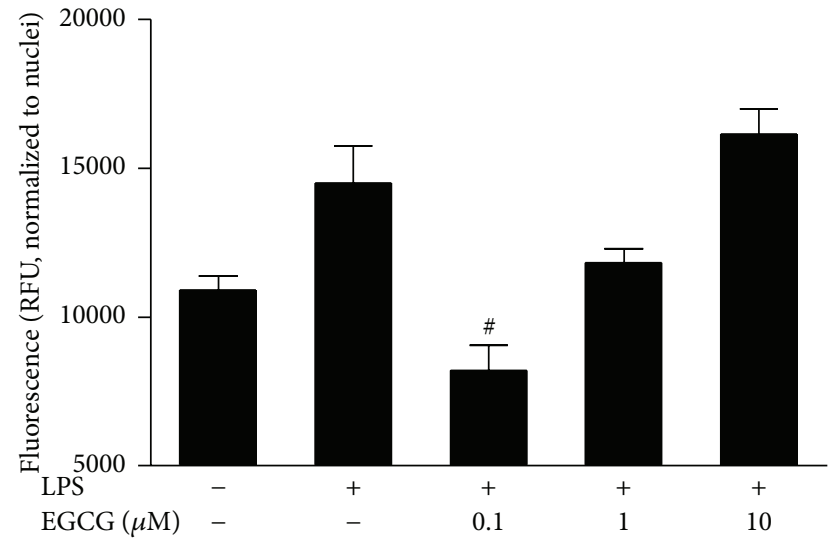

(b)

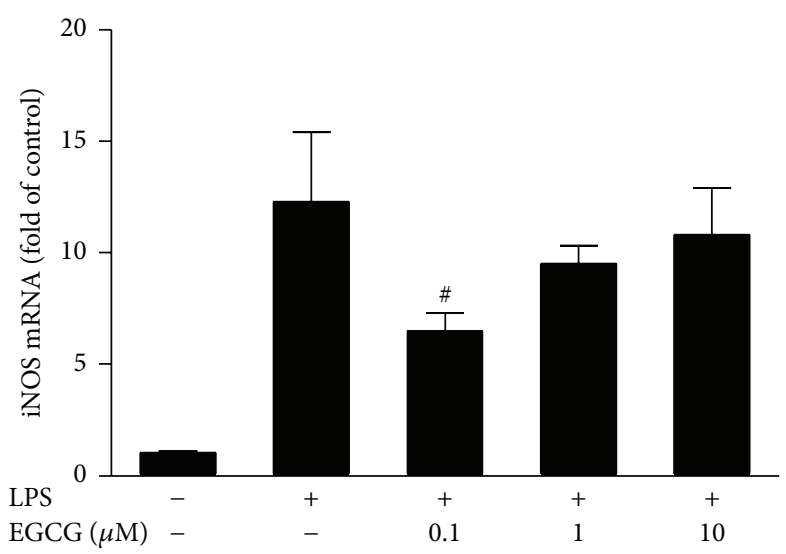

(c)

FIGURE 6: Effect of EGCG on LPS-induced production of ROS in neurons. Primary human neuronal cultures were treated with or without LPS at indicated concentrations for $48 \mathrm{~h}$ (a) or with indicated concentrations of EGCG for $1 \mathrm{~h}$ prior to $100 \mathrm{ng} / \mathrm{mL}$ of LPS treatment for additional $48 \mathrm{~h}$ (b). The ROS production was examined by labelling cells with a cell-permeable nonfluorescent probe $2^{\prime}, 7^{\prime}$-dichlorofluorescin diacetate and the fluorescence was measured by a fluorescence microplate reader with excitation at $488 \mathrm{~nm}$ and emission at $527 \mathrm{~nm}$. (c) The expression of iNOS in neurons treated with LPS in the presence of indicated concentration of EGCG. Data were expressed as mean \pm SD of three independent experiments. ${ }^{*} P<0.05$, as compared with control; ${ }^{*} P<0.05$, as compared with LPS treated.

effect at least partially attributed to the antioxidant activity of EGCG at this concentration. This biphasic mode of action implicates that EGCG may be a good candidate for treatment of inflammation-associated neurodegenerative disorders given the limited availability of EGCG to the brain. Nevertheless, further studies with oral administration of EGCG to suitable animal model are needed.

\section{Competing Interests}

The authors declare that they have no competing interests.

\section{Authors' Contributions}

Jie-Liang Li and Wen-Zhe Ho conceived of the study and experiments and wrote the paper. Jie-Liang Li, Jin-Biao Liu, Yi-Zhong Wang, Li Zhou, Xu Wang, and Yu Zhou performed experiments. All authors have read and approved the final version of this paper.

\section{Acknowledgments}

This work was supported by the National Institutes of Health (Grants DA027550, DA036163, and DA022177 to Wen-Zhe Ho; DA036413 and DA040329 to Jie-Liang Li) and the National Natural Science Foundation of China (81271334 to Wen-Zhe Ho). HIV/AIDS Research Award from the Robert Mapplethorpe Foundation to Jie-Liang Li is also acknowledged.

\section{References}

[1] J. Gehrmann, Y. Matsumoto, and G. W. Kreutzberg, "Microglia: intrinsic immuneffector cell of the brain," Brain Research Reviews, vol. 20, no. 3, pp. 269-287, 1995.

[2] M. L. Block, L. Zecca, and J.-S. Hong, "Microglia-mediated neurotoxicity: uncovering the molecular mechanisms," Nature Reviews Neuroscience, vol. 8, no. 1, pp. 57-69, 2007.

[3] A. Yadav and R. G. Collman, "CNS inflammation and macrophage/microglial biology associated with HIV-1 
infection," Journal of Neuroimmune Pharmacology, vol. 4, no. 4, pp. 430-447, 2009.

[4] E. H. Byun, Y. Fujimura, K. Yamada, and H. Tachibana, "TLR4 signaling inhibitory pathway induced by green tea polyphenol epigallocatechin-3-gallate through $67-\mathrm{kDa}$ laminin receptor," Journal of Immunology, vol. 185, no. 1, pp. 33-45, 2010.

[5] Y. Nakamura, Q. S. Si, and K. Kataoka, "Lipopolysaccharideinduced microglial activation in culture: temporal profiles of morphological change and release of cytokines and nitric oxide," Neuroscience Research, vol. 35, no. 2, pp. 95-100, 1999.

[6] H.-M. Gao, J. Jiang, B. Wilson, W. Zhang, J.-S. Hong, and B. Liu, "Microglial activation-mediated delayed and progressive degeneration of rat nigral dopaminergic neurons: relevance to Parkinson's disease," Journal of Neurochemistry, vol. 81, no. 6, pp. 1285-1297, 2002.

[7] Z. Ling, Y. Zhu, C. W. Tong, J. A. Snyder, J. W. Lipton, and P. M. Carvey, "Progressive dopamine neuron loss following supranigral lipopolysaccharide (LPS) infusion into rats exposed to LPS prenatally," Experimental Neurology, vol. 199, no. 2, pp. 499512, 2006.

[8] L. Qin, Y. Liu, X. Qian, J.-S. Hong, and M. L. Block, "Microglial NADPH oxidase mediates leucine enkephalin dopaminergic neuroprotection," Annals of the New York Academy of Sciences, vol. 1053, pp. 107-120, 2005.

[9] L. Qin, Y. Liu, T. Wang et al., "NADPH oxidase mediates lipopolysaccharide-induced neurotoxicity and proinflammatory gene expression in activated microglia," Journal of Biological Chemistry, vol. 279, no. 2, pp. 1415-1421, 2004.

[10] A. Lampron, A. Elali, and S. Rivest, "Innate immunity in the CNS: redefining the relationship between the CNS and its environment," Neuron, vol. 78, no. 2, pp. 214-232, 2013.

[11] G. Gras and M. Kaul, "Molecular mechanisms of neuroinvasion by monocytes-macrophages in HIV-1 infection," Retrovirology, vol. 7, article 30, 2010.

[12] S. J. Schachtele, S. Hu, M. R. Little, and J. R. Lokensgard, "Herpes simplex virus induces neural oxidative damage via microglial cell Toll-like receptor-2," Journal of Neuroinflammation, vol. 7, article 35, 2010.

[13] A. Scalbert, C. Manach, C. Morand, C. Rémésy, and L. Jiménez, "Dietary polyphenols and the prevention of diseases," Critical Reviews in Food Science and Nutrition, vol. 45, no. 4, pp. 287306, 2005.

[14] H.-C. Ou, T.-Y. Song, Y.-C. Yeh et al., "EGCG protects against oxidized LDL-induced endothelial dysfunction by inhibiting LOX-1-mediated signaling," Journal of Applied Physiology, vol. 108, no. 6, pp. 1745-1756, 2010.

[15] H. Xu, W. T. Lui, C. Y. Chu, P. S. Ng, C. C. Wang, and M. S. Rogers, "Anti-angiogenic effects of green tea catechin on an experimental endometriosis mouse model," Human Reproduction, vol. 24, no. 3, pp. 608-618, 2009.

[16] H. Y. Ahn, Y. Xu, and S. T. Davidge, "Epigallocatechin-3-Ogallate inhibits TNF $\alpha$-induced monocyte chemotactic protein1 production from vascular endothelial cells," Life Sciences, vol. 82, no. 17-18, pp. 964-968, 2008.

[17] H. Lee, J. H. Bae, and S.-R. Lee, "Protective effect of green tea polyphenol EGCG against neuronal damage and brain edema after unilateral cerebral ischemia in gerbils," Journal of Neuroscience Research, vol. 77, no. 6, pp. 892-900, 2004.

[18] B. A. Sutherland, O. M. Shaw, A. N. Clarkson, D. M. Jackson, I. A. Sammut, and I. Appleton, "Neuroprotective effects of (-)epigallocatechin gallate following hypoxia-ischemia-induced brain damage: novel mechanisms of action," The FASEB Journal, vol. 19, no. 2, pp. 258-260, 2005.

[19] O. Kucera, V. Mezera, A. Moravcova et al., "In vitro toxicity of epigallocatechin gallate in rat liver mitochondria and hepatocytes," Oxidative Medicine and Cellular Longevity, vol. 2015, Article ID 476180, 10 pages, 2015.

[20] Y. Levites, T. Amit, S. Mandel, and M. B. Youdim, "Neuroprotection and neurorescue against Abeta toxicity and PKCdependent release of nonamyloidogenic soluble precursor protein by green tea polyphenol (-)-epigallocatechin-3-gallate," The FASEB Journal, vol. 17, pp. 952-954, 2003.

[21] G.-X. Li, Y.-K. Chen, Z. Hou et al., "Pro-oxidative activities and dose-response relationship of (-)-epigallocatechin-3-gallate in the inhibition of lung cancer cell growth: a comparative study in vivo and in vitro," Carcinogenesis, vol. 31, no. 5, pp. 902-910, 2010.

[22] R. C. C. de Pace, X. Liu, M. Sun et al., "Anticancer activities of (-)-epigallocatechin-3-gallate encapsulated nanoliposomes in MCF7 breast cancer cells," Journal of Liposome Research, vol. 23, no. 3, pp. 187-196, 2013.

[23] S. Hu, W. S. Sheng, J. R. Lokensgard, and P. K. Peterson, "Morphine induces apoptosis of human microglia and neurons," Neuropharmacology, vol. 42, no. 6, pp. 829-836, 2002.

[24] J. Li, L. Ye, D. R. Cook et al., "Soybean-derived Bowman-Birk inhibitor inhibits neurotoxicity of LPS-activated macrophages," Journal of Neuroinflammation, vol. 8, article 15, 2011.

[25] D. J. Loane, A. Kumar, B. A. Stoica, R. Cabatbat, and A. I. Faden, "Progressive neurodegeneration after experimental brain trauma: association with chronic microglial activation," Journal of Neuropathology and Experimental Neurology, vol. 73, no. 1, pp. 14-29, 2014.

[26] M. E. Lull and M. L. Block, "Microglial activation and chronic neurodegeneration," Neurotherapeutics, vol. 7, no. 4, pp. 354$365,2010$.

[27] X.-G. Luo, J.-Q. Ding, and S.-D. Chen, "Microglia in the aging brain: relevance to neurodegeneration," Molecular Neurodegeneration, vol. 5, article 12, 2010.

[28] S. V. More, H. Kumar, I. S. Kim, S.-Y. Song, and D.-K. Choi, "Cellular and molecular mediators of neuroinflammation in the pathogenesis of Parkinson's disease," Mediators of Inflammation, vol. 2013, Article ID 952375, 12 pages, 2013.

[29] H. Wang, S. Zhu, R. Zhou, W. Li, and A. E. Sama, “Therapeutic potential of HMGB1-targeting agents in sepsis," Expert Reviews in Molecular Medicine, vol. 10, article e32, 2008.

[30] K. U. Tufekci, S. Genc, and K. Genc, “The endotoxin-induced neuroinflammation model of Parkinson's disease," Parkinson's Disease, vol. 2011, Article ID 487450, 25 pages, 2011.

[31] T. H. Mogensen, "Pathogen recognition and inflammatory signaling in innate immune defenses," Clinical Microbiology Reviews, vol. 22, no. 2, pp. 240-273, 2009.

[32] A. Cagnin, D. J. Brooks, A. M. Kennedy et al., "In-vivo measurement of activated microglia in dementia," The Lancet, vol. 358, no. 9280, pp. 461-467, 2001.

[33] P. Eikelenboom, C. Bate, W. A. Van Gool et al., "Neuroinflammation in Alzheimer's disease and prion disease," GLIA, vol. 40, no. 2, pp. 232-239, 2002.

[34] P. L. McGeer and E. G. McGeer, "Inflammatory processes in amyotrophic lateral sclerosis," Muscle and Nerve, vol. 26, no. 4, pp. 459-470, 2002.

[35] P. T. Nelson, L. A. Soma, and E. Lavi, "Microglia in diseases of the central nervous system," Annals of Medicine, vol. 34, no. 7-8, pp. 491-500, 2002. 
[36] E.-H. Byun, T. Omura, K. Yamada, and H. Tachibana, "Green tea polyphenol epigallocatechin-3-gallate inhibits TLR2 signaling induced by peptidoglycan through the polyphenol sensing molecule 67-kDa laminin receptor," FEBS Letters, vol. 585, no. 5, pp. 814-820, 2011.

[37] M. Menegazzi, E. Tedeschi, D. Dussin et al., "Anti-interferon gamma action of epigallocatechin-3-gallate mediated by specific inhibition of STAT1 activation," The FASEB Journal, vol. 15, no. 7, pp. 1309-1311, 2001.

[38] B. J. M. Ripley, M. Fujimoto, S. Serada et al., "Green tea polyphenol epigallocatechin gallate inhibits cell signaling by inducing SOCS1 gene expression," International Immunology, vol. 22, no. 5, pp. 359-366, 2010.

[39] J. Li, L. Ye, X. Wang et al., “(-)-Epigallocatechin gallate inhibits endotoxin-induced expression of inflammatory cytokines in human cerebral microvascular endothelial cells," Journal of Neuroinflammation, vol. 9, article 161, 2012.

[40] N. Ahmad, S. Gupta, and H. Mukhtar, "Green tea polyphenol epigallocatechin-3-gallate differentially modulates nuclear factor $\kappa \mathrm{B}$ in cancer cells versus normal cells," Archives of Biochemistry and Biophysics, vol. 376, no. 2, pp. 338-346, 2000.

[41] Y. Cai, S.-S. Yu, T.-T. Chen et al., "EGCG inhibits CTGF expression via blocking NF- $\kappa$ B activation in cardiac fibroblast," Phytomedicine, vol. 20, no. 2, pp. 106-113, 2013.

[42] R. Li, Y.-G. Huang, D. Fang, and W.-D. Le, “(-)Epigallocatechin gallate inhibits lipopolysaccharide-induced microglial activation and protects against inflammationmediated dopaminergic neuronal injury," Journal of Neuroscience Research, vol. 78, no. 5, pp. 723-731, 2004.

[43] B. Zhang, B. Wang, S. Cao, and Y. Wang, "Epigallocatechin-3gallate (EGCG) attenuates traumatic brain injury by inhibition of edema formation and oxidative stress," The Korean Journal of Physiology \& Pharmacology, vol. 19, no. 6, pp. 491-497, 2015.

[44] Y. Levites, O. Weinreb, G. Maor, M. B. H. Youdim, and S. Mandel, "Green tea polyphenol (-)-epigallocatechin-3-gallate prevents N-methyl-4-phenyl- 1,2,3,6-tetrahydropyridine-induced dopaminergic neurodegeneration," Journal of Neurochemistry, vol. 78, no. 5, pp. 1073-1082, 2001.

[45] K. Rezai-Zadeh, G. W. Arendash, H. Hou et al., "Green tea epigallocatechin-3-gallate (EGCG) reduces $\beta$-amyloid mediated cognitive impairment and modulates tau pathology in Alzheimer transgenic mice," Brain Research, vol. 1214, pp. 177187, 2008.

[46] K. Rezai-Zadeh, D. Shytle, N. Sun et al., "Green tea epigallocatechin-3-gallate (EGCG) modulates amyloid precursor protein cleavage and reduces cerebral amyloidosis in Alzheimer transgenic mice," The Journal of Neuroscience, vol. 25, no. 38, pp. 8807-8814, 2005.

[47] T. Itoh, M. Imano, S. Nishida et al., “(-)-Epigallocatechin-3gallate increases the number of neural stem cells around the damaged area after rat traumatic brain injury," Journal of Neural Transmission, vol. 119, no. 8, pp. 877-890, 2012.

[48] T. Itoh, M. Tabuchi, N. Mizuguchi et al., "Neuroprotective effect of (-)-epigallocatechin-3-gallate in rats when administered preor post-traumatic brain injury," Journal of Neural Transmission, vol. 120, no. 5, pp. 767-783, 2013.

[49] M. Suganuma, S. Okabe, M. Oniyama, Y. Tada, H. Ito, and H. Fujiki, "Wide distribution of $[3 \mathrm{H}](-)$-epigallocatechin gallate, a cancer preventive tea polyphenol, in mouse tissue," Carcinogenesis, vol. 19, no. 10, pp. 1771-1776, 1998.

[50] K. Nakagawa and T. Miyazawa, "Absorption and distribution of tea catechin, (-)-epigallocatechin-3-gallate, in the rat," Journal of Nutritional Science and Vitaminology, vol. 43, no. 6, pp. 679684, 1997.

[51] K. Herges, J. M. Millward, N. Hentschel, C. Infante-Duarte, O. Aktas, and F. Zipp, "Neuroprotective effect of combination therapy of glatiramer acetate and epigallocatechin-3-gallate in neuroinflammation," PLOS ONE, vol. 6, no. 10, Article ID e25456, 2011.

[52] C. Hu and D. D. Kitts, "Evaluation of antioxidant activity of epigallocatechin gallate in biphasic model systems in vitro," Molecular and Cellular Biochemistry, vol. 218, no. 1-2, pp. 147155, 2001.

[53] O. Weinreb, T. Amit, S. Mandel, and M. B. H. Youdim, "Neuroprotective molecular mechanisms of (-)-epigallocatechin-3gallate: a reflective outcome of its antioxidant, iron chelating and neuritogenic properties," Genes \& Nutrition, vol. 4, no. 4, pp. 283-296, 2009.

[54] O. Weinreb, S. Mandel, and M. B. H. Youdim, "cDNA gene expression profile homology of antioxidants and their antiapoptotic and proapoptotic activities in human neuroblastoma cells," The FASEB Journal, vol. 17, no. 8, pp. 935-937, 2003.

[55] O. Weinreb, S. Mandel, and M. B. H. Youdim, "Gene and protein expression profiles of anti- and pro-apoptotic actions of dopamine, R-apomorphine, green tea polyphenol (-)epigallocatechine-3-gallate, and melatonin," Annals of the New York Academy of Sciences, vol. 993, pp. 351-393, 2003.

[56] L. Elbling, R.-M. Weiss, O. Teufelhofer et al., "Green tea extract and (-)-epigallocatechin-3-gallate, the major tea catechin, exert oxidant but lack antioxidant activities," The ASEB Journal, vol. 19, no. 7, pp. 807-809, 2005.

[57] Y.-T. Szeto and I. F. F. Benzie, "Effects of dietary antioxidants on human DNA ex vivo," Free Radical Research, vol. 36, no. 1, pp. 113-118, 2002.

[58] A. Furukawa, S. Oikawa, M. Murata, Y. Hiraku, and S. Kawanishi, "(-)-Epigallocatechin gallate causes oxidative damage to isolated and cellular DNA," Biochemical Pharmacology, vol. 66, no. 9, pp. 1769-1778, 2003.

[59] S. Oikawa, A. Furukawa, H. Asada, K. Hirakawa, and S. Kawanishi, "Catechins induce oxidative damage to cellular and isolated DNA through the generation of reactive oxygen species," Free Radical Research, vol. 37, no. 8, pp. 881-890, 2003.

[60] B. A. Sutherland, R. M. A. Rahman, and I. Appleton, "Mechanisms of action of green tea catechins, with a focus on ischemia-induced neurodegeneration," Journal of Nutritional Biochemistry, vol. 17, no. 5, pp. 291-306, 2006.

[61] B. Halliwell, "Vitamin C: antioxidant or pro-oxidant in vivo?" Free Radical Research, vol. 25, no. 5, pp. 439-454, 1996. 


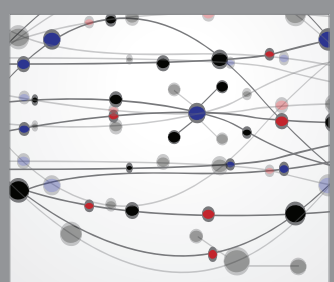

The Scientific World Journal
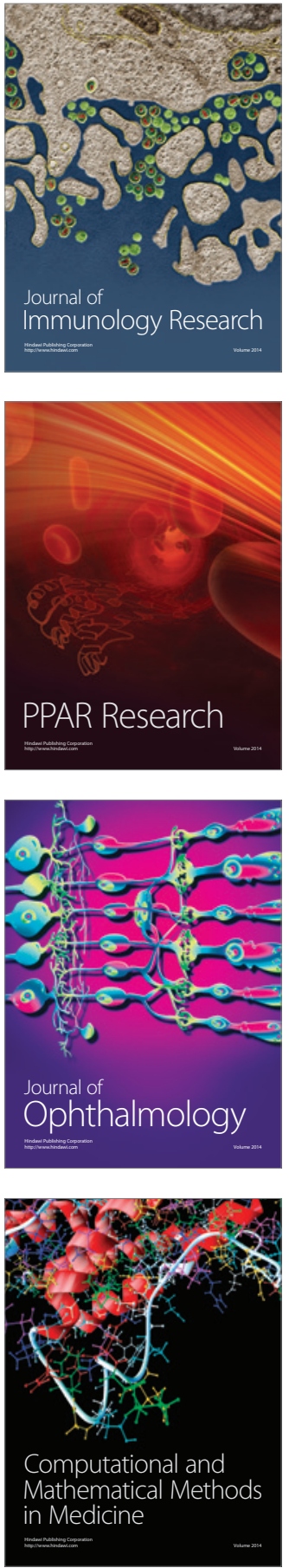

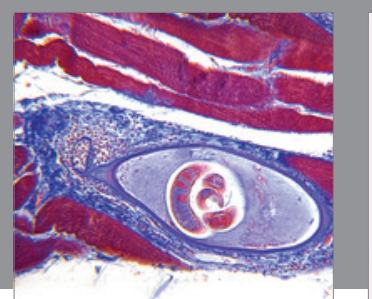

Gastroenterology Research and Practice

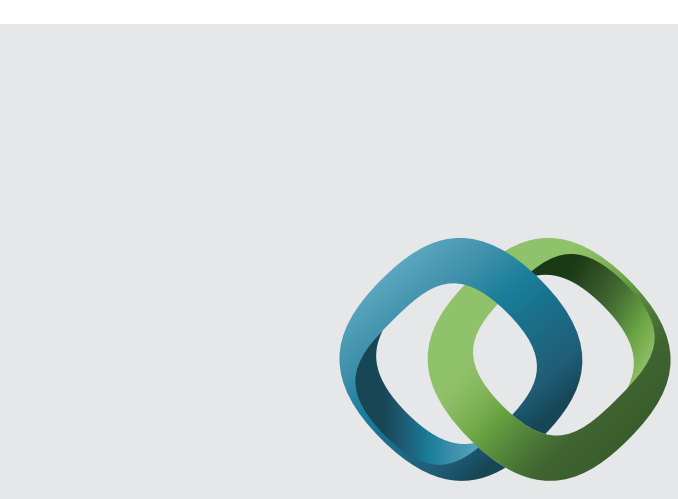

\section{Hindawi}

Submit your manuscripts at

http://www.hindawi.com
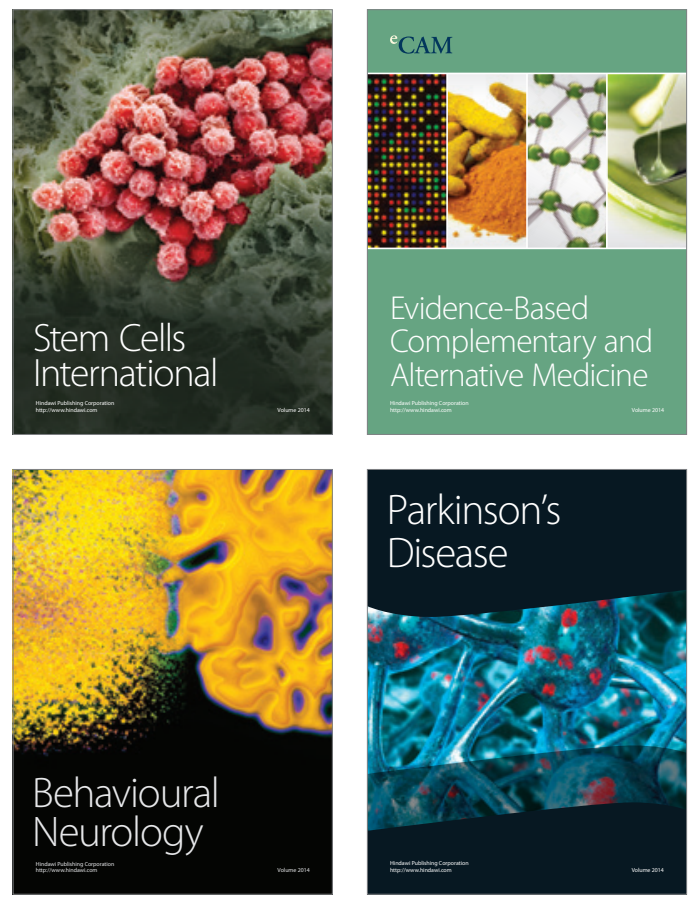
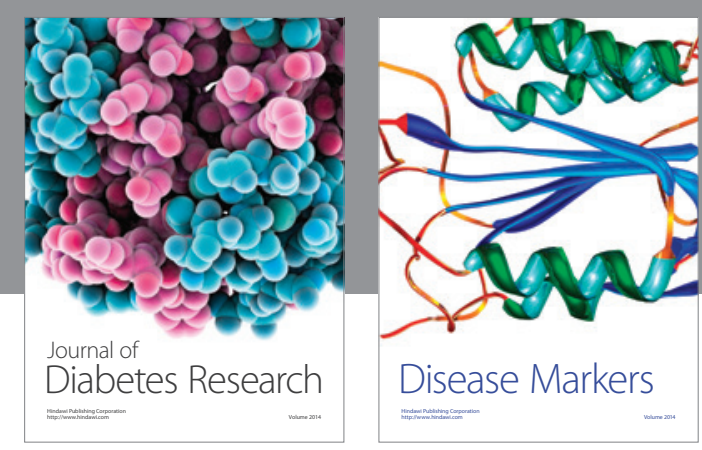

Disease Markers
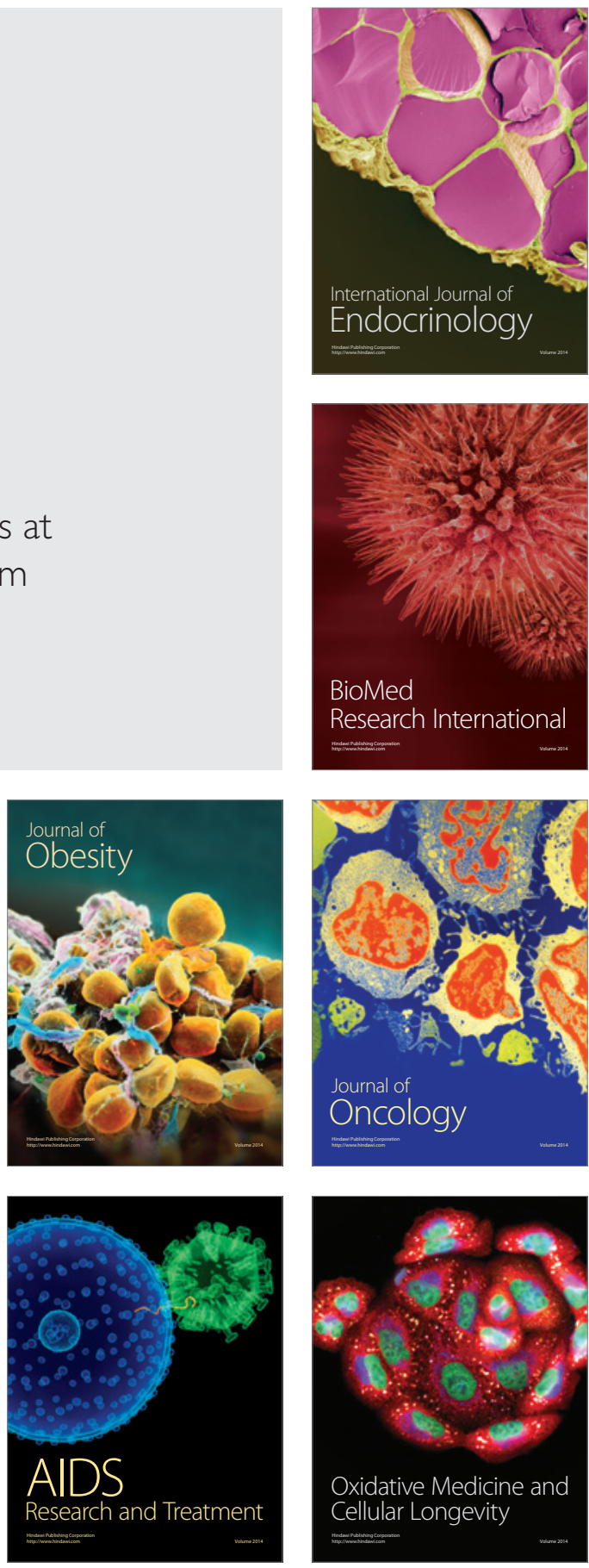